\title{
Evaluating appropriate maximum holding times for private well water samples
}

\author{
Allison Maier, ${ }^{*}$ Julia Krolik, ${ }^{*}$ Stephanie Fan, ${ }^{\dagger}$ Patricia Quintin, ${ }^{*}$ Danielle McGolrick, $^{\dagger}$ Alan Joyce, ${ }^{*}$ and \\ Anna Majury*, ${ }^{*}, \xi, \uparrow$ \\ *Public Health Ontario, 181 Barrie St., Kingston, ON \\ ${ }^{\dagger}$ Department of Biomedical and Molecular Sciences, Queen's University, Kingston, ON \\ Department of Applied Science, St. Lawrence College, Kingston, ON \\ ${ }^{\S}$ Department of Pathology and Molecular Medicine, Queen's University, Kingston, ON \\ 'Department of Public Health Sciences, Queen's University, Kingston, ON
}

\begin{abstract}
Privately maintained groundwater wells are often located at a great distance from laboratories, creating a barrier to bacteriological testing (a necessity for determining drinking water potability). Extending the acceptable holding time between testing and collection could potentially diminish this barrier. Using seven Escherichia coli strains isolated from private well waters, the acceptability of Ontario's current allowable sample maximum holding time $(48 \mathrm{~h})$ was compared with time of collection. Additionally, the acceptability of extending the holding time from $48 \mathrm{~h}$ to $72 \mathrm{~h}$ was investigated. All analyses were performed using noninferiority statistical approaches to determine if later holding times had no meaningful difference in bacterial growth (determined by colony forming unit enumeration). All strains did not statistically decrease below the acceptable 10\% difference during the two time periods. However, variations in the survival rates of isolates were observed, suggesting that a risk management approach should be employed when determining maximum holding times.
\end{abstract}

Key words: holding times, well water, Escherichia coli.

Routine bacteriological testing of drinking water is essential as it provides an indication of water potability. In most North American jurisdictions, privately maintained water supplies, predominantly groundwater wells, are the responsibility of the owner and testing is not regulated. Most of these wells are located rurally; thus, travel time from the site of the well to the laboratory may be significant and a potential barrier to testing for some private well owners. As such, the maximum acceptable holding time between the collection and testing of the water sample is a vital consideration for well owner populations.

Currently, there are inconsistent maximum acceptable holding times globally: the United States Environmental Protection Agency (EPA) allows a maximum of $30 \mathrm{~h}$ for groundwater samples (US Environmental Protection Agency 2008), whereas Health Canada states $24 \mathrm{~h}$ is acceptable ( $8 \mathrm{~h}$ is preferred), though up to $48 \mathrm{~h}$ may be allowable for remote areas, but these recommendations are not specific for wells (Health Canada 2012). The World Health Organization's drinking water recommendations are also not specific to groundwater and state that $6 \mathrm{~h}$ is ideal and $24 \mathrm{~h}$ is the maximum allowable (World Health Organization 2003). Furthermore, there is limited and inconsistent scientific

Corresponding author: Anna Majury (email: anna.majury@oahpp.ca) evidence supporting these policies, none of which is specific to groundwater samples. Previous studies have investigated surface water (lakes and rivers, often those receiving municipal or agricultural waste) (Standridge and Lesar 1977; Dutka and El-Shaarawi 1980; Pope et al. 2003; Aulenbach 2010), municipal and industrial effluent (Standridge and Lesar 1977; Dutka and El-Shaarawi 1980; Selvakumar et al. 2004), stormwater (Selvakumar et al. 2004), and water from within municipal distribution systems (McDaniels et al. 1985). Survival rates were variable for different fecal indicator bacteria (FIB), with total coliforms often decreasing shortly after collection (Mendez Novelo et al. 2010), whereas fecal coliforms generally survived longer, possibly up to $62 \mathrm{~h}$ (Aulenbach 2010). Some studies observed significant decreases in Escherichia coli (E. coli) density by 18-24 h (Selvakumar et al. 2004, Aulenbach 2010), while other studies suggested that up to $48 \mathrm{~h}$ was acceptable (Pope et al. 2003). Notably, across all of these studies, there was very little power to analyze holding times greater than $48 \mathrm{~h}$.

In Ontario, testing of samples from private water supplies is available through the Public Health Ontario Laboratories (PHOLs). Well owners collect water samples and are instructed to keep them cold (refrigerated at approximately $4^{\circ} \mathrm{C}$, but not frozen) until they can drop off the sample directly at one of 11 
laboratories located across the province or at a drop off location operated by local health units (typically at their offices or municipal offices) (Public Health Ontario 2014). The PHOLs are licensed to provide testing of well water samples by the Ontario Ministry of the Environment and Climate Change (MOECC), whose testing protocols are used by all licensed laboratories. According to these MOECC protocols, samples must be tested within $48 \mathrm{~h}$ of collection (MOECC 2012), and are often rejected for being "too old" because of various delays between the time of collection and laboratory testing, as seen Figure 1. Prior to 2012, the PHOLs allowed for samples to be accepted for up to two days (i.e., a maximum of 56 h) instead of the stricter two day definition of $48 \mathrm{~h}$ - this protocol change resulting in, on average, an additional 1500 rejected submissions. Once samples arrive at the laboratory, bacteriological analysis is performed using membrane filtration and differential coliform enumeration for both E. coli and total coliform colony forming units (CFUs).

The limitations posed by the $48 \mathrm{~h}$ window are particularly problematic given that earlier work reveals very low levels of testing among well owners; a recent five-year review of well water submissions in southeastern Ontario suggested that over 99\% of wells are not tested according to local guidelines (three samples without evidence of bacterial contamination each year) (Maier et al. 2014). This is further corroborated by a survey conducted in southern Ontario, where 65\% of well owners stated that they tested their water less than once a year (Kreutzwiser et al. 2008). Previous research suggests that many owners do not test because of inconvenience, including the strict $48 \mathrm{~h}$ requirement (Jones et al. 2006; Imgrund et al. 2011). As such, increasing the acceptable maximum holding time might increase the rate of testing. This, in turn, would increase the number of well owners who are making informed decisions regarding well water consumption based on bacteriological potability.

To address the lack of groundwater-specific evidence available to inform policy development, a study was designed to evaluate existing protocols. Current and potential maximum acceptable holding times for $E$. coli enumeration were evaluated: specifically $(i)$ the current practice in Ontario of $48 \mathrm{~h}$ as compared with initial collection and (ii) extending the $48 \mathrm{~h}$ to $72 \mathrm{~h}$.

\section{Methods and rationale}

\section{Study design}

The study was designed to simulate the effects of holding times on enumeration of $E$. coli in private well water samples, utilizing wild type strains (in this context, bacteria which were cultured directly from environmental samples as compared to standard laboratory strains). Five wild type E. coli were isolated from well waters submitted to the Kingston PHOL (WT-K1 through WT-K5). Efforts were undertaken to ensure wild types were isolated across multiple seasons. To test the geographic generalizability of the results, two wild types were isolated at different PHOLs (one from Hamilton (WT-H) and Orillia (WT-OR) respectively).

Three holding times were considered within this analysis: $0 \mathrm{~h}$ (to represent the well water sample as first collected), $48 \mathrm{~h}$ (the current maximum holding time allowed by the MOECC), and $72 \mathrm{~h}$ (an additional $24 \mathrm{~h}$ period after the current maximum holding time). To simulate the conditions between collection and testing (advised to be between $2^{\circ} \mathrm{C}$ and $8^{\circ} \mathrm{C}$ ), samples were stored in a refrigerator at $4^{\circ} \mathrm{C}$.

All testing was performed utilizing a well water matrix to simulate the sample environment. The matrix was obtained by autoclaving well water, which was pooled from incoming private well water submissions, and for which prior testing produced a "no growth" result. The matrix consisted of 10-15 samples representing local yet geographically diverse wells.

Plate counting methods have an inherently high variability (International Organization for Standardization 2000). To minimize the effects of this, plating was performed in triplicate. During design, data normality of all plate counts was

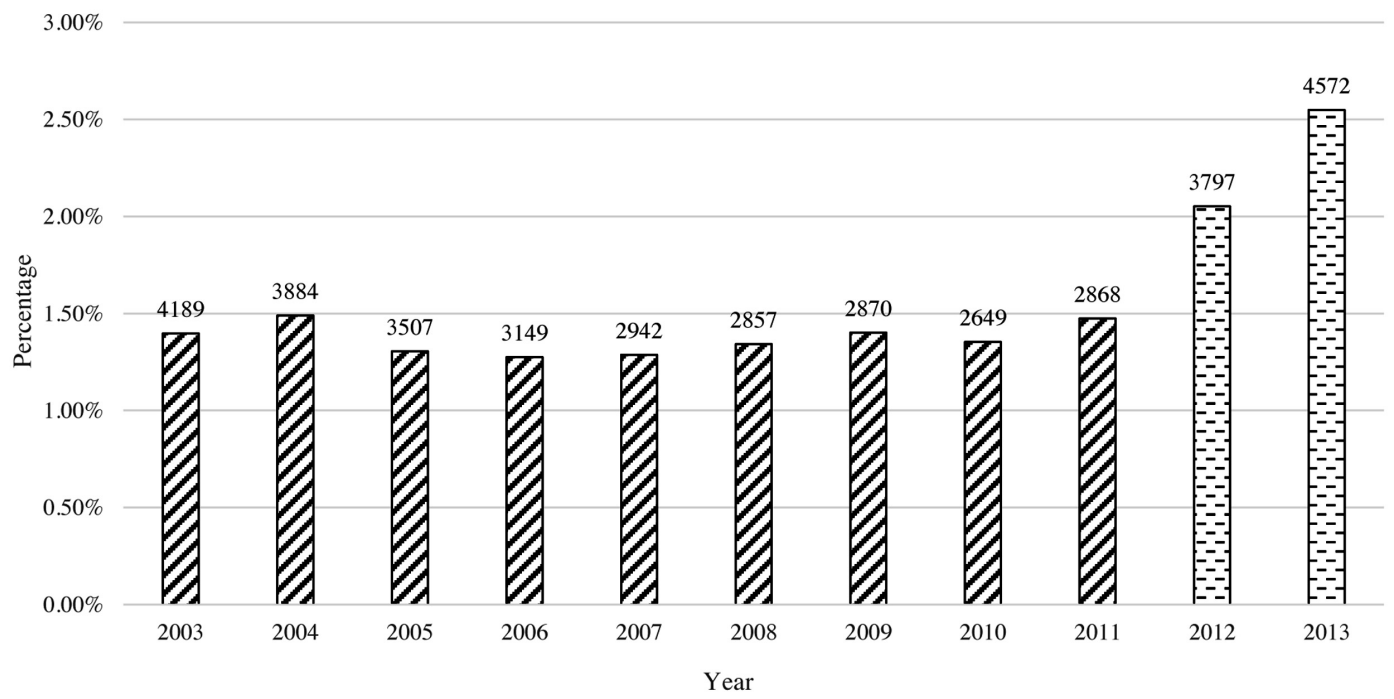

Fig. 1. Percentage and number of samples rejected for being "too old" by the testing laboratory (PHOL) from 20032013, inclusive (unpublished data, Majury 2013). 
confirmed. Subsequently, means of each triplicate set were used for analysis. Additionally, each wild type was tested by a single analyst (whose reproducibility had been previously confirmed) as between-person variability is known to be an issue (International Organization for Standardization 2000). In total, only two separate analysts took part in this study to minimize, but not discount entirely, variability. Finally, to account for the high variability (and therefore the high variance in the data), a sufficient sample size $(n=25)$ was chosen for statistical analysis.

\section{Laboratory methods}

The wild type presumptive $E$. coli isolates were confirmed as E. coli using API E20 strips (Biomérieux, Durham, NC). For each trial conducted, E. coli was cultured on Columbia blood agar with $5 \%$ sheep blood (Oxoid, Nepean, ON) and incubated at $37^{\circ} \mathrm{C}$ for $18-24 \mathrm{~h}$. A bacterial suspension, using the well water matrix, was created to an optical density of 1 at $600 \mathrm{~nm}$ (with an acceptable range between 0.950 and 1.049) using the NanoDrop 2000c (Thermo Scientific, DE) (henceforth known as an OD1 suspension). It was previously determined (through testing 10-fold serial dilution from 10 to $10^{-7}$ ) that $10^{-6}$ dilutions resulted in growth between the suggested range for counting (25-250 colonies per plate) (Sutton 2011). Therefore, for each wild type, 25 OD1 suspensions were created and subsequently serially diluted to $10^{-6}$, plated in triplicate, incubated at $37^{\circ} \mathrm{C}$ for $18-24 \mathrm{~h}$, and enumerated. A schematic diagram depicting the process is shown in Figure 2.

\section{Statistical analysis}

Mean CFU counts (CFUCs) (of the 25 triplicate means) were determined for each strain and holding time, and normality for each was checked using both skewness and kurtosis methods. Additionally, any outliers were identified and removed where they affected normality. These analyses were performed using IBM SPSS Statistics 22 (IBM Corp., Armonk, NY). The means were subsequently graphed for observational purposes.

As the goal of this study was to evaluate the validity of the current maximum holding time, including whether it could be increased from $48 \mathrm{~h}$ to $72 \mathrm{~h}$, it was necessary to determine that the CFUCs did not decrease over time. Traditional statistical methods (employed by the majority of holding time literature
(Dutka and El-Shaarawi 1980; McDaniels et al. 1985; Pope et al. 2003; Selvakumar et al. 2004; Aulenbach 2010)) prove significant differences between means of groups by rejecting the null hypothesis (equivalent means) in favour of an alternate hypothesis (the means are different) (Streiner 2003). However, in these methods, failing to disprove the null (an insignificant result) is not equivalent to proving the null hypothesis is true (Mascha and Sessler 2011). A new methodology, which aims to test for equivalence has been developed for clinical purposes (i.e., proving two therapies have equivalent outcomes) and can be applied here. More specifically noninferiority testing, a onesided test similar to equivalence that is used to determine that one treatment is no worse on the outcome measure than the original (Mascha and Sessler 2011), can be used prove that mean CFUCs over multiple holding times have not decreased. These statistical methods reverse the traditional null and alternate hypotheses and again prove the alternate hypothesis by rejecting the null. However, as variability is inherent in the data, it is not possible to prove two means are exactly the same and instead the tests focus on trivial versus nontrivial differences (i.e., differences that have a practical/clinical impact). Specifically, for noninferiority testing, the new mean $\left(\mu_{1}\right)$ should be no lower than the original $\left(\mu_{0}\right)$ and the null hypothesis becomes (Equation 1):

$\mu_{1}-\mu_{0} \leq-\delta$

where $\delta$ is the value where the difference becomes meaningful as determined by practice.

In this case the alternate hypothesis is (Equation 2):

$\mu_{1}-\mu_{0}>-\delta$

which is interpreted as the new mean is better or at least no more than $\delta$ worse than the original (Mascha and Sessler 2011).

For this study, the meaningful difference was determined to be $10 \%$ of the original mean CFUC. This was based on the probability of misclassifying water samples as either positive or negative for $E$. coli (i.e., a $10 \%$ difference represents one in 10 $E$. coli cells dying and a $10 \%$ probability of misclassifying a water sample that originally contained only one E. coli CFU and a $1 \%$ probability of misclassifying a water sample that originally contained two E. coli CFUs).

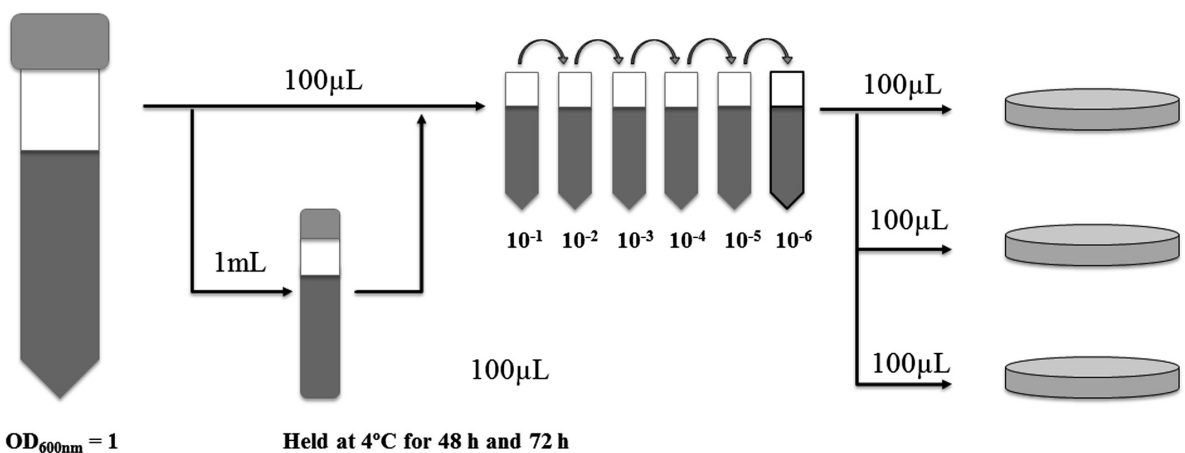

Fig. 2. Diagram of testing process. 


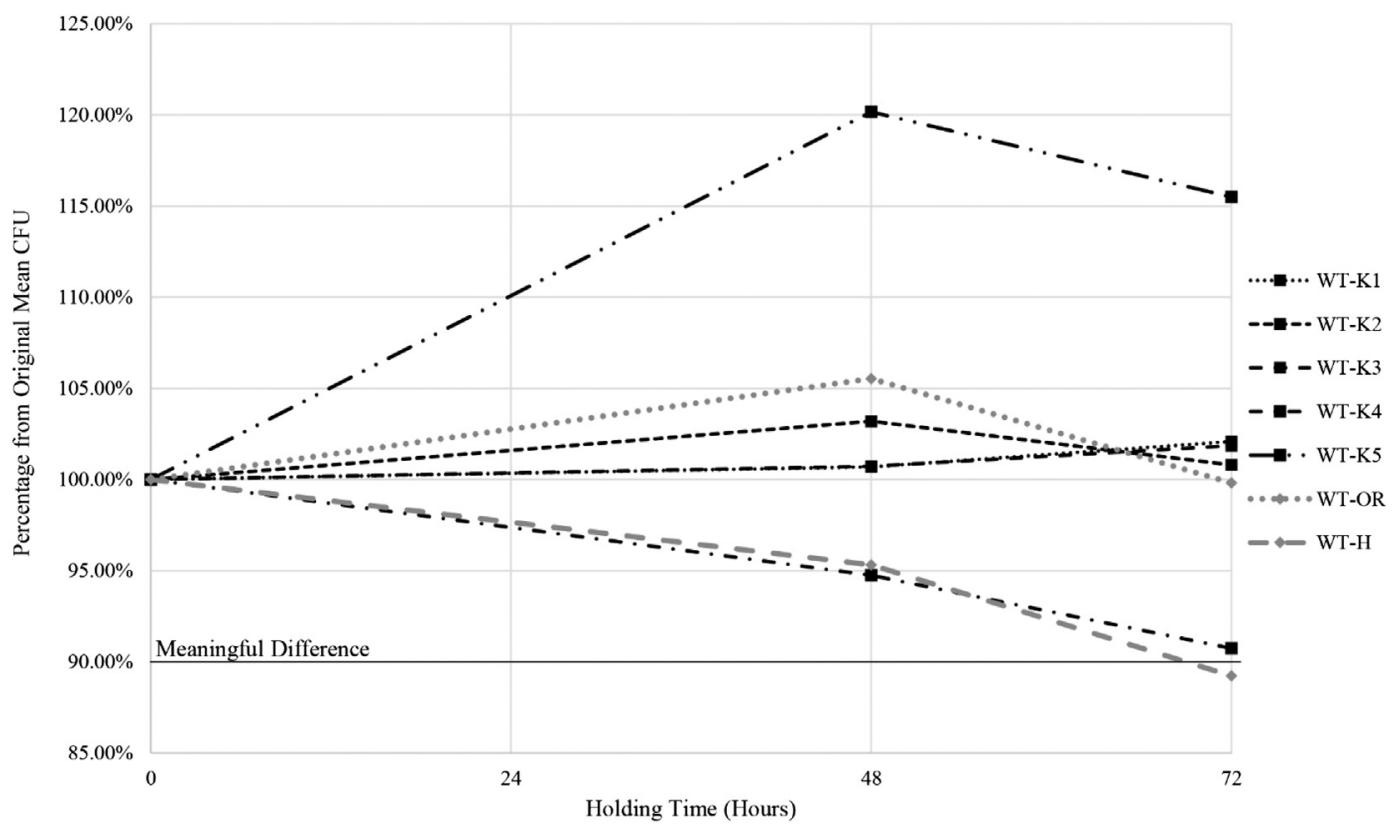

Fig. 3. Change in mean CFUC for the seven strains at $48 \mathrm{~h}$ and $72 \mathrm{~h}$, as a percentage of $0 \mathrm{~h}$ mean CFUC.

To meet the two goals of the study (to compare $0 \mathrm{~h}$ and $48 \mathrm{~h}$ and then $48 \mathrm{~h}$ and $72 \mathrm{~h}$ ), noninferiority tests compared mean CFUCs at $0 \mathrm{~h}$ with $48 \mathrm{~h}$, and separately mean CFUCs at $48 \mathrm{~h}$ and 72 h. For each analysis, the $\delta$ was determined to be $10 \%$ of the mean CFUC at the lower time period. Given that the means were found from the same OD1 suspension held for different times, a paired $t$-test design was utilized. The paired $t$-test formula $(t)$, adjusted for the noninferiority test, is (Equation 3):

$t=\frac{\bar{d}-\delta}{(S d / \sqrt{N})}$

where degrees of freedom $=n-1$

The test is one-sided and statistical noninferiority was defined as a $p \leq 0.05$.

\section{Results}

The skewness and kurtosis values for mean CFUCs for all strains were acceptable, and therefore the data were normal. One strain (WT-H) had two outliers removed, resulting in a final sample size of 23 (due to the repeated measures nature, an outlier at one time period resulted in the OD1 suspension being removed across all time periods).

Figure 3 shows the mean CFUCs over time for the strains as a percentage of the original mean for comparison across strains. Strains are grouped by colour to represent their origin (Kingston PHOL (black) vs. other PHOL (grey)). The $10 \%$ decrease determined to be the meaningful difference for this study is overlaid on the graph. The actual mean CFU counts (and standard deviation $(\sigma)$ ) for each strain at each time period can be seen in Table 1 .
Table 1. Mean CFUC and standard deviation $(\sigma)$ for each strain at each time period.

\begin{tabular}{|c|c|c|c|c|c|c|c|}
\hline \multirow{2}{*}{$\begin{array}{l}\text { Wild } \\
\text { type }\end{array}$} & \multirow[b]{2}{*}{ No. } & \multicolumn{2}{|c|}{ O hs } & \multicolumn{2}{|c|}{$48 \mathrm{~h}$} & \multicolumn{2}{|c|}{$72 \mathrm{~h}$} \\
\hline & & Mean & $\sigma$ & Mean & $\sigma$ & Mean & $\sigma$ \\
\hline WT-K1 & 25 & 94.4 & 11.7 & 95.0 & 10.5 & 96.3 & 7.0 \\
\hline WT-K2 & 25 & 90.9 & 8.1 & 93.8 & 9.6 & 91.6 & 10.2 \\
\hline WT-K3 & 25 & 90.5 & 10.3 & 91.1 & 8.3 & 92.1 & 10.3 \\
\hline WT-K4 & 25 & 119.9 & 7.8 & 113.6 & 9.0 & 108.8 & 9.3 \\
\hline WT-K5 & 25 & 72.8 & 5.8 & 87.5 & 8.2 & 84.1 & 6.2 \\
\hline WT-H & 23 & 97.7 & 5.0 & 93.1 & 5.18 & 87.1 & 7.4 \\
\hline WT-OR & 25 & 129.3 & 9.9 & 136.5 & 9.8 & 129.1 & 7.7 \\
\hline
\end{tabular}

All wild types were tested for noninferiority (with $10 \%$ of the original mean CFUC being designated as the meaningful difference). The results of these tests are presented in Table 2. The results show that both objectives regarding no meaningful difference across holding times were met. Specifically, no statistical inferiority was found for all wild types between $(i)$ $0 \mathrm{~h}$ and $48 \mathrm{~h}$ and (ii) $48 \mathrm{~h}$ and $72 \mathrm{~h}$. This would suggest the current policy of $48 \mathrm{~h}$ is acceptable, but also that it would be equally acceptable to extend the maximum allowable holding time to $72 \mathrm{~h}$, as no meaningful decrease in CFUCs was observed for any of the wild types tested.

However, when combined with observational results this conclusion is weaker. Five wild types do appear to remain close to $100 \%$ of the original after $72 \mathrm{~h}$, with some wild types having increasing mean CFUCs by $48 \mathrm{~h}$ and (or) $72 \mathrm{~h}$. However, two wild types do show a general downward trend from $0 \mathrm{~h}$ to $48 \mathrm{~h}$ to $72 \mathrm{~h}$. This suggests that behavioural variability exists within 
Table 2. Statistical results from noninferiority paired $t$-tests.

\begin{tabular}{|c|c|c|c|c|c|c|c|c|}
\hline \multirow[b]{2}{*}{ Wild type } & \multicolumn{4}{|c|}{$0 \mathrm{~h}$ to $48 \mathrm{~h}$ comparison } & \multicolumn{4}{|c|}{$48 \mathrm{~h}$ to $72 \mathrm{~h}$ comparison } \\
\hline & $10 \%$ difference (CFUC) & df & $t$ & $P$ & $10 \%$ difference (CFUC) & df & $t$ & $P$ \\
\hline WT-K1 & 9.4 & 24 & 5.0 & $<0.01$ & 9.5 & 24 & 5.9 & $<0.01$ \\
\hline WT-K2 & 9.1 & 24 & 5.1 & $<0.01$ & 9.4 & 24 & 2.5 & 0.01 \\
\hline WT-K3 & 9.1 & 24 & 8.3 & $<0.01$ & 9.1 & 24 & 5.7 & $<\mathbf{0 . 0 1}$ \\
\hline WT-K4 & 12.0 & 24 & 3.1 & $<\mathbf{0 . 0 1}$ & 11.4 & 24 & 3.4 & $<\mathbf{0 . 0 1}$ \\
\hline WT-K5 & 7.3 & 24 & 11.2 & $<0.01$ & 8.7 & 24 & 3.0 & $<0.01$ \\
\hline WT-H & 9.8 & 22 & 3.2 & $<\mathbf{0 . 0 1}$ & 9.3 & 22 & 1.8 & 0.04 \\
\hline WT-OR & 12.9 & 24 & 8.8 & $<0.01$ & 13.6 & 24 & 2.66 & 0.01 \\
\hline
\end{tabular}

Note: $D f$ is the degree of freedom of the test. Statistically significant results are in bold.

survival rates amongst wild types and that the appropriateness of either $48 \mathrm{~h}$ or $72 \mathrm{~h}$ holding times may be questionable.

\section{Discussion}

This study was a simulation of $E$. coli survival rates in well waters; that is, not an exact replica of laboratory procedures for testing well water samples. Given variability of the underlying phenomenon and the complexity in measuring, the study design has some limitations. For example, most of the well water samples received for testing at the laboratory have less than $10 \mathrm{E}$. coli CFUs, but this range is not quantitative (International Organization for Standardization 2000) and so a higher concentration was necessary for the study. These higher concentrations might have an impact (positively or negatively) on survival rates, making it challenging to generalize the findings to lower concentrations, though a study by Dutka and El-Shaarawi (1980) provided evidence that bacterial density does not appear to be a factor in survival rates of water samples.

Overall, the generalizability of these results may be limited. Although a sufficient within wild type sample size was utilized for statistical purposes, the high time and resource requirements of the study only allowed for seven wild types to be included, all of which were from southern Ontario. Furthermore, the confirmed E. coli wild types were chosen at random (with some control for season); therefore, very little is known about them. As such, they may not adequately represent all wild types in the region and may not be generalizable to other regions. Additionally, the high resource demand limited the number of holding time periods that could be reasonably analyzed by the study. There may be key points in time where survival rates change (either between $0 \mathrm{~h}$ and $48 \mathrm{~h}$, between $48 \mathrm{~h}$ and $72 \mathrm{~h}$, or after $72 \mathrm{~h}$ ); if so, these would be the most appropriate maximum holding times. Finally, only E. coli was modelled in this study, though the PHOL, like many other laboratories, also reports total coliform CFUs, which may behave differently than E. coli. However, an E. coli result is considered to be evidence of fecal contamination of water, whereas total coliforms are only considered to be evidence of bacterial contamination, so $E$. coli was the preferable indicator to model.

Contradictory results regarding the holding of E. coli for $48 \mathrm{~h}$ have been found by earlier studies (Pope et al. 2003; Selvakumar et al. 2004). No previous investigations have had the power to analyze a $72 \mathrm{~h}$ holding time. Furthermore, comparison between the results of this study and previous studies is challenging for two major reasons: $(i)$ different source waters and (ii) different statistical approaches. As evidenced by this study and previous literature, considerable variability exists within survival rates of $E$. coli and other FIB (notably total coliforms) from similar water sources, across multiple sites (i.e., different rivers or lakes, effluents from different facilities, or in the case of this study different isolated wild types). Given the variability within similar water sources, it is inadvisable to extrapolate these results across multiple water sources (for example surface water compared with groundwater). As such, the evidence presented by this study provides new insight into possible acceptable holding times that are specific for groundwater. Additionally, most studies employ traditional statistical methods (determining statistically significant differences) to develop survival models for FIB to evaluate holding times. However, as noted previously, these approaches do not have the capability of determining if CFUCs are meaningfully the same across possible holding times. Therefore, this paper provides a novel statistical framework for evaluating acceptable holding times for all water sources.

Both of the holding times evaluated by this study are longer than the maximum holding time utilized by many jurisdictions, including the EPA. The $72 \mathrm{~h}$ holding time is longer than the current maximum allowable holding time according to the MOECC. Given the persuasive, albeit limited, results of this study (and the limited current body of literature), a risk management approach should be employed when determining interim protocols as well as investment in further research to support development of evidence-based policies. Specifically, maximum acceptable holding times must balance the risk between: (i) the likelihood of reporting a false negative due to decreased survival after an extended holding time with (ii) the 
rates of specimens not tested due to rejection or not submitted due to owners' inability to submit a sample on time. Additionally, although this study was focused on private well waters, increasing the acceptable maximum holding times for well water samples from public systems may also have an impact. Rural and (or) remote community systems, which are at a great distance from the closest certified laboratory, may currently find it difficult and (or) cost-prohibitive to test well water samples regularly. Increasing the acceptable holding time might allow these systems greater access to testing.

This was a time- and resource-consuming study; to test the seven wild types across three time periods required 175 OD1 suspensions, resulting in over 1500 plates used. Further investigation is required to fully evaluate acceptable holding times, notably examining a larger geographic region, various FIB, and additional holding times, to fully substantiate the most appropriate window.

\section{Acknowledgements}

The authors would like to acknowledge all the employees at the Public Health Ontario Laboratories, especially Kayla Poirier and Sandra Edelsward.

\section{References}

Aulenbach, B.T. 2010. Bacteria holding times for fecal coliform by $\mathrm{mFC}$ agar method and total coliform and Escherichia coli by Colilert-18 Quanti-Tray method. Environ Monit Assess, 161: 147-159. PMID: 19165613. doi: 10.1007/s10661-008-0734-3.

Dutka, B.J., and El-Shaarawi, A. 1980. Microbiological water and effluent sample preservation. Can J Microbiol, 26(8): 921-929. PMID: 7006769. doi: 10.1139/m80-159.

Health Canada. 2012. Guidelines for Canadian drinking water quality: guideline technical document - total coliforms. [Online] Available: http://www.hc-sc.gc.ca/ewh-semt/alt_formats/hecs-sesc/ pdf/pubs/water-eau/coliforms-coliformes/coliforms-coliformes-eng. pdf [Accessed 14 May 2014].

Imgrund, K., Kreutzwiser, R., and de Loë, R. 2011. Influences on the water testing behaviors of private well owners. J Water Health, 9(2): 241-252. PMID: 21942190. doi: 10.2166/wh.2011.139.

International Organization for Standardization. 2000. Water quality Guidance on validation of microbiological methods-ISO/TR 13843. Switzerland.

Jones, A.Q., Dewey, C.E., Dore, K., Majowisz, S.E., McEwan, S.A., Waltner-Toews, D., Eric, M., Carr, D.J., and Henson, S.J. 2006. Public perceptions of drinking water: a postal survey of residents with private water supplies. BMC Public Health, 6: 94. doi: $10.1186 / 1471-2458-6-94$.
Kreutzwiser, R., de Loë, R.C., and Imgrund, K. 2008. Out of sight, out of mind: private water well stewardship in Ontario - report on the findings of the Ontario household water well survey. Waterloo, ON: Water Policy and Governance Group, University of Water.

Maier, A., Krolik, J., Randhawa, K., and Majury, A. 2014. Bacteriological testing of private well water: a trends and guidelines assessment using five years of submissions data from southeastern Ontario. Can J Public Health, 105(3): e203-e208. PMID: 25165840 .

Mascha, E.J., and Sessler, D.I. 2011. Equivalence and noninferiority testing in regression models and repeated-measures designs. Anesth Analg, 112(3): 678-687. PMID: 21304155. doi: 10.1213/ANE. 0b013e318206f872.

McDaniels, A.E., Bordner, R.H., Gartside, P.S., Haines, J.R., Brenner, K.P., and Rankin, C.C. 1985. Holding effects on coliform enumeration in drinking water samples. Appl Environ Microbiol, 50(2): 755-762. PMID: 4083877.

Mendez Novelo, R.I., San Pedro Cedillo, L., Castillo Borges, E.R., and Vazquez Borges, E. 2010. Modeling of holding time of water biological samples. Rev Int Contam Ambient, 26(4): 327-335.

Ontario Ministry of the Environment. 2012. Membrane filtration method using DC Agar for the simultaneous detection and enumeration of total coliforms and Escherichia Coli in drinking water - MICROMFDC-E3407. Toronto, ON.

Pope, M.L., Bussen, M., Feige, M.A., Shadix, L., Gonder, S., Rodgers, C., Chambers, Y., Pulz, J., Miller, K., Connell, K., and Standridge, J. 2003. Assessment of the effects of holding time and temperature on Escherichia Coli densities in surface water samples. Appl Environ Microbiol, 69(10): 6201-6207. PMID: 14532081. doi: 10.1128/AEM.69.10.6201-6207.2003.

Public Health Ontario. 2014. Water Testing. [Online] Available: http://www.publichealthontario.ca/en/ServicesAndTools/Laboratory Services/Pages/Water-testing.aspx\#.U34AQ3JdWy4 [Accessed 22 May 2014].

Selvakumar, A., Borst, M., Boner, M., and Mallon, P. 2004. Effects of sample holding time on concentrations of microorganisms in water samples. Water Environ Res, 76(1): 67-72. PMID: 15058466. doi: $10.2175 / 106143004 X 141591$.

Standridge, J.H., and Lesar, D.J. 1977. Comparison of four-hour and twenty-four-hour refrigerated storage of nonpotable water for fecal coliform analysis. Appl Environ Microbiol, 34(4): 398-402.

Streiner, D.L. 2003. Unicorns Do Exist: a tutorial on "proving" the null hypothesis. Can J Psychiatry, 48(11): 756-761.

Sutton, S. 2011. Accuracy of plate counts. J Validation Technol, 17(3): 42-46.

US Environmental Protection Agency. 2008. Analytical methods approved for compliance monitoring under the ground water rule. Washington, DC.

World Health Organization. 2003. Assessing microbial safety of drinking water. Cornwall: TJ International Ltd. 